\title{
Sepsis-associated encephalopathy
}

\author{
Teneille E. Gofton and G. Bryan Young
}

\begin{abstract}
Sepsis-associated encephalopathy (SAE) is a diffuse brain dysfunction that occurs secondary to infection in the body without overt CNS infection. SAE is frequently encountered in critically ill patients in intensive care units, and in up to $70 \%$ of patients with severe systemic infection. The severity of SAE can range from mild delirium to deep coma. Seizures and myoclonus are infrequent and cranial nerves are almost always spared, but most severe cases have an associated critical illness neuromyopathy. Development of SAE probably involves a number of mechanisms that are not mutually exclusive and vary from patient to patient. Substantial neurological and psychological morbidities often occur in survivors. Mortality is almost always due to multiorgan failure rather than neurological complications, and is almost $70 \%$ in patients with severe SAE. Further research into the pathophysiology, management and prevention of SAE is needed. This Review discusses the epidemiology and clinical presentation of SAE. Recent evidence for SAE pathophysiology is outlined and a diagnostic approach to patients with this syndrome is presented. Lastly, prognosis and management of SAE is discussed.
\end{abstract}

Gofton, T. E. \& Young, G. B. Nat. Rev. Neurol. 8, 557-566 (2012); published online 18 September 2012; doi:10.1038/nrneurol.2012.183

\section{Introduction}

Sepsis-associated encephalopathy (SAE) is commonly seen in systemically ill patients. The syndrome is defined by diffuse cerebral dysfunction that accompanies sepsis in the absence of direct CNS infection, structural abnormality or other types of encephalopathy (for example, hepatic or renal encephalopathy), as detected by clinical or standard laboratory tests. Patients presenting with SAE show evidence of severe systemic infection with features of sepsis or systemic inflammatory response syndrome (SIRS). ${ }^{1}$ SAE manifests as a spectrum of disturbed cerebral function ranging from mild delirium to coma. As mortality is increased with severity of SAE, ${ }^{2}$ early identification and management of patients with SAE are important to reduce associated morbidity and mortality. Delirium is often the first manifestation of sepsis, providing a useful diagnostic clue. SAE should trigger a search for infection and prompt initiation of appropriate therapy.

Cerebral dysfunction seen in SAE reflects the systemic metabolic, inflammatory and haemodynamic disturbances that are associated with SIRS, rather than a direct CNS abnormality. SAE is effectively a diagnosis of exclusion, which can be made after meningitis, encephalitis, septic emboli from endocarditis and noninfective systemic inflammation (as seen in pancreatitis, burns or trauma) have been ruled out. Despite lack of direct CNS infection, laboratory evidence of CNS dysfunction is common in SAE and may manifest as abnormalities in EEG measurements, somatosensory evoked potentials, levels of biomarkers of CNS injury (such as neuronspecific enolase [NSE] and protein S100b), and in results of neuroradiological tests. ${ }^{3}$ None of these manifestations

Competing interests

The authors declare no competing interests. is specific for SAE, and SAE diagnosis depends on the clinical context and the presence of evidence for an infection somewhere in the body.

The implications of sepsis and SIRS on cerebral function are profound, and the body of knowledge regarding SAE is growing. Further research, however, is clearly necessary to improve recovery from SAE and reduce long-term consequences on cerebral function. This article provides an overview of current knowledge on the epidemiology and clinical presentation of SAE. We review recent evidence for the pathophysiology of SAE and present a diagnostic approach to patients with this disease. Lastly, prognosis of SAE and a management strategy for the disorder are discussed.

\section{Epidemiology}

Sepsis is a SIRS that accompanies an identified or suspected infection-a definition established by Bone and colleagues. ${ }^{4}$ A group of experts attending the International Sepsis Definitions Conference in 2001 defined SIRS as a syndrome presenting with two or more of the following symptoms: increased or decreased body temperature $\left(>38^{\circ} \mathrm{C}\right.$ or $\left.<36^{\circ} \mathrm{C}\right)$, hyperventilation (respiratory rate $>20$ breaths per min or an arterial partial pressure of carbon dioxide $<32 \mathrm{mmHg}$ ) and abnormal white blood cell count $(>12,000$ or $<4,000$ cells $/ \mu \mathrm{l}){ }^{5}$ Severe sepsis is defined as sepsis together with organ dysfunction and hypoperfusion or hypotension, ${ }^{4}$ and septic shock is defined as sepsis together with arterial hypotension despite appropriate fluid resuscitation. ${ }^{4}$ Patients with sepsis and encephalopathy were previously diagnosed as having septic encephalopathy. However, this term has fallen out of favour, because it suggests an active infection within the CNS.
Departments of Clinical Neurological Sciences and Medicine, London Health Sciences Centre, University Hospital, University of Western Ontario, 339 Windermere Road, London, ON N6A 5A5, Canada (T. E. Gofton, G. B. Young).

Correspondence to: G. B. Young

bryan.young@ lhsc.on.ca 


\section{Key points}

- Sepsis-associated encephalopathy (SAE) is an early feature of infection in the body and might appear before other systemic features of sepsis are obvious

- SAE has a spectrum of degrees of severity, ranging from delirium to deep coma

- Hyperventilation can be an early feature and paratonic rigidity might be the only neurological finding, besides delirium, in early SAE; advanced disease is associated with critical illness polyneuropathy in $70 \%$ of cases

- No specific markers for SAE exist, so diagnosis relies on exclusion of primary CNS infection and other causes of encephalopathy

- Although early stages of SAE might have a uniform pathogenesis, multiple mechanisms, which can operate alone or in combination, could be involved in advanced disease

- Morbidity and mortality increase with disease severity; early investigation and prompt treatment of underlying infection when delirium presents are, therefore, important
SAE had significantly higher heart rate, blood lactate and serum sodium levels, as well as lower platelet count, serum albumin levels and serum $\mathrm{pH} .{ }^{2}$ The source and the aetiology of infection are also important factors: biliary tract or intestinal infections are associated with greater risk of SAE, and the most commonly implicated organisms include Staphylococcus aureus, Enterococcus feacium, Acinetobacter spp., Pseudomonas aeruginosa and Stenotrophomonas maltophilia. ${ }^{2}$ This study has limitations including a small sample size and being a retrospective analysis, but the exclusion criteria were rigorous, which ensured that patients with encephalopathy of alternative aetiologies were excluded from the analysis. Overall, it seems that patients with advancedstage SAE have a higher burden of systemic illness than those with early-stage disease, and that the source of infection and causative organism can be important in the development of SAE. and prevalence of SAE. ${ }^{1,2}$ The studies are varied and most do not have a uniform study cohort. Indeed, even the operational definition of SAE is problematic, as patients might develop encephalopathy in the early phases of infection without meeting the criteria for sepsis, or a confused state can develop in patients with a remote infection, such as an abdominal, walled-off abscess that is not 'septic'. One of the challenges in studying SAE is that without a specific diagnostic test, it remains a syndrome diagnosed by exclusion.

SAE is thought to be the most common cause of encephalopathy in the medical-surgical intensive care unit (ICU), ${ }^{6}$ and over half of patients with sepsis have encephalopathy. ${ }^{7}$ Incidence of encephalopathy is higher in patients who have bacteraemia and evidence of renal, hepatic or multiorgan failure. As sepsis is a leading cause of ICU admissions and $20-50 \%$ of patients with sepsis have delirium, SAE is highly prevalent in the ICU. ${ }^{8} 70 \%$ of patients with bacteraemia have neurological symptoms ranging from lethargy to coma, and $>80 \%$ have abnormalities on EEG. ${ }^{9,10}$ Approximately half (46\%) of patients with bacteraemia have SAE. ${ }^{1,9}$ Patients with acutely altered mental status associated with encephalopathy have higher mortality rates (49\%) than patients with pre-existing mental status changes (41\%) or normal mental status (26\%). ${ }^{11}$ A recent international study (the Delirium Epidemiology in Critical Care study) involving 497 patients in ICU showed that the prevalence of delirium was $32.3 \%$ and that sepsis was the leading cause of medical illnesses that required ICU admission. ${ }^{12}$

In another study from China, Zhang et al. ${ }^{2}$ retrospectively reviewed patients admitted to ICU over a 3-year period. The exclusion criteria included pre-existing or chronic liver or kidney failure, severe electrolyte imbalances, blood glucose disturbances, CNS infections or pre-existing CNS disease, previous cardiac arrest with resuscitation or previous treatment with sedative drugs. Out of 323 patients, 41 had SAE and the rest had sepsis without encephalopathy. This analysis demonstrated that patients with higher APACHE II (Acute Physiology and Chronic Health Evaluation II) scores and lower scores on the Glasgow coma scale (GCS) are more likely to have SAE. ${ }^{2}$ Compared with patients without SAE, those with

\section{Clinical presentation and diagnosis}

SAE is one of many causes of delirium. Patients with SAE can be expected to present with a level of consciousness that is out of keeping with the degree of any sedative treatment they are receiving, and they might show disturbances of sleep-wake cycles or evidence of hallucinations, restlessness or agitation, among other symptoms commonly seen in delirium. Apart from the abnormal mental status and gegenhalten (paratonic rigidity), the findings in the neurological examination are unremarkable. However, $70 \%$ of advanced cases of SAE have an associated critical illness neuromyopathy. ${ }^{7}$ With advanced-stage SAE, cranial nerve function is invariably spared, ${ }^{13}$ which is a useful factor in the differential diagnosis of Guillain-Barré syndrome. Hyperventilation in SAE is often due to respiratory alkalosis in the early, delirious phase and to metabolic acidosis in advanced-stage sepsis. ${ }^{14}$ As patients with SAE have sepsis by definition, pulse rate is also commonly, but not invariably, elevated. ${ }^{5}$

SAE is often suspected in patients presenting with acutely altered mental status accompanied by sepsis or septic shock (Figure 1). When evaluating a patient with sepsis and delirium, the primary goal should be to investigate and eliminate the possibility of a primary CNS pathology that might cause delirium. Some common primary neurological disturbances that can lead to delirium include cerebral abscess, encephalitis, meningitis, stroke, nonconvulsive status epilepticus or, more rarely, primary CNS vasculitis. ${ }^{15,16}$ Frequent confounding factors, such as sedative medications, can make evaluation of mental status challenging.

Patients suspected of having SAE should undergo a thorough investigation through clinical history, medication review, physical examination, laboratory tests, neuroimaging and EEG to assess anatomical abnormalities, bearing in mind that criteria for sepsis might not yet be met (Box 1), but aiming to determine whether the patient has an infection. The clinical history should include a search for evidence of prior CNS pathology, hepatic or renal dysfunction and prior episodes of 
delirium associated with severe illness or recent infections. A detailed review of medications may provide information on recent or discontinued medications, such as benzodiazepines, other psychoactive agents or recent antibiotics, which might cause encephalopathy or delirium. A history of overdose of or withdrawal from psychoactive medications might suggest an alternative aetiology for reduced level of consciousness or delirium, and a recent history of infection requiring antibiotics can help to pinpoint a source of sepsis, and thereby lead to diagnosis of SAE. A thorough physical examination should be done to investigate a source of infection (for example, infection of a decubitus ulcer or other types of cellulitis or rash), to assess the evidence for organ dysfunction (for example, scleral icterus and jaundice suggest hepatic dysfunction) and to identify any focal neurological abnormalities, including hemiparesis or cranial nerve abnormalities, which might suggest a focal neurological process, such as an abscess or a stroke. Importantly, the clinician should attend promptly to any changes in the mental status of the patient, especially to problems associated with attention or concentration.

Multiple screening tools for detection of delirium are available, but none is specific for SAE. For practical purposes, we advocate using the Confusion Assessment Method (CAM) for patients outside the ICU. The CAM has recently been validated for use in diagnosis of delirium in this setting and shows excellent sensitivity (94-100\%) and positive predictive value (91-94\%)..$^{17,18}$ However, the version of the CAM that was developed for ICU patients (CAM-ICU) had only about $41-47 \%$ sensitivity when used by regular ICU nurses, ${ }^{19,20}$ although the specificity was excellent (98\%). Patients with hypoactive delirium are most likely to be missed with the CAM. Unstructured delirium assessments or the Intensive Care Delirium Screening Checklist seem to have greater sensitivity for detecting delirium in the ICU than does the CAM, but outcomes of patients missed with the CAM-ICU seem to be similar to those of general ICU patients. ${ }^{21}$ Thus, the most appropriate delirium screening tool for ICU remains controversial. The use of coma scales in SAE has not been explored, but the FOUR (Full Outline of UnResponsiveness) scale-a clinical grading scale used for assessing level of consciousness-is more suitable for intubated ICU patients than is the GCS. ${ }^{22}$ Nonetheless, the GCS can be useful in predicting the course of SAE.?

No specific biomarker exists for SAE and it remains largely a clinical diagnosis. However, a myriad of abnormalities are seen in patients with SAE, including abnormalities in laboratory tests that do no directly assess neurological function and, by definition, patients have sepsis. A comprehensive metabolic panel of analyses including a complete blood count, measurement of expanded electrolytes (sodium, potassium, chloride, magnesium, phosphate and calcium) and serum enzyme levels (alanine and aspartate aminotransferases, alkaline phosphatase and $\gamma$-glutamyltransferase), renal function tests (serum creatinine), and investigations for infection (blood cultures, respiratory or sputum culture,

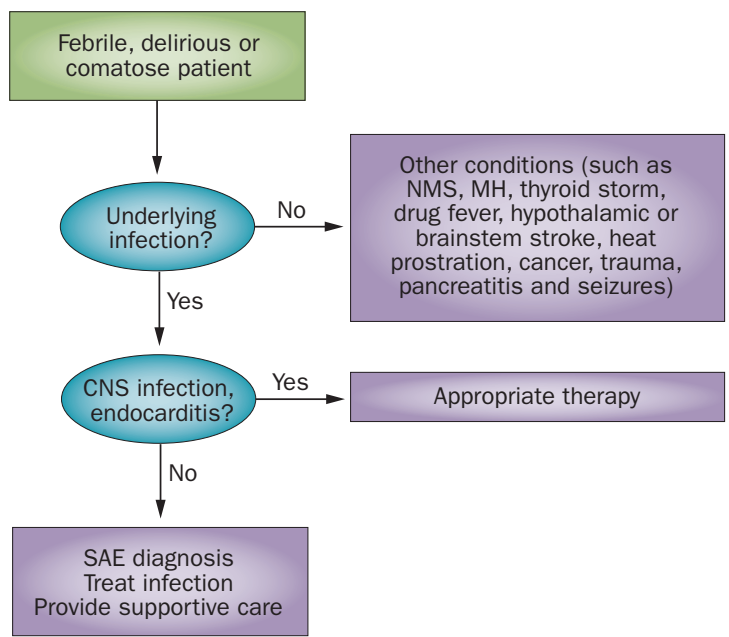

Figure 1 | Diagnostic approach to patients showing symptoms of SAE. A systematic approach helps to avoid missing other potential contributors to delirium. Abbreviations: $\mathrm{MH}$, malignant hyperthermia; NMS, neuroleptic malignant syndrome; SAE, sepsis-associated encephalopathy.

Box 1 | Laboratory and pathological features of SAE
Neuroimaging
Normal
Multiple ischaemic strokes
Nonspecific subcortical white matter lesions
Cerebrospinal fluid analysis
Elevated protein levels
Normal glucose levels and cell culture analysis
EEG recordings
Normal
Theta waves
Delta waves
Triphasic waves
Burst-suppression pattern
Pathological findings
Minimal to no abnormalities
Subtle neuronal drop-out
Cerebral ischaemia
Haemorrhages
Multifocal necrotizing leukoencephalopathy
Microabscesses
Abbreviation: SAE, sepsis-associated encephalopathy.

urine culture, and cerebrospinal fluid [CSF] analysis and culture) are required. Evidence of bacteraemia or other haematological infection should also be sought.

The absence of bacteraemia does not exclude SAE, and identification of a pathological organism is not always possible. Common reasons for difficulty in identifying a disease-causing organism include prior antibiotic use, which might hinder detection of specific organisms, or presence of an occult abscess. By definition, evidence of CNS infection in the CSF rules out a diagnosis of SAE. However, elevations of CSF protein levels are expected and are thought to occur as a result of increased permeability of the blood-brain barrier $(\mathrm{BBB})^{10}$ and movement of proteins from blood into the CSF. 
a

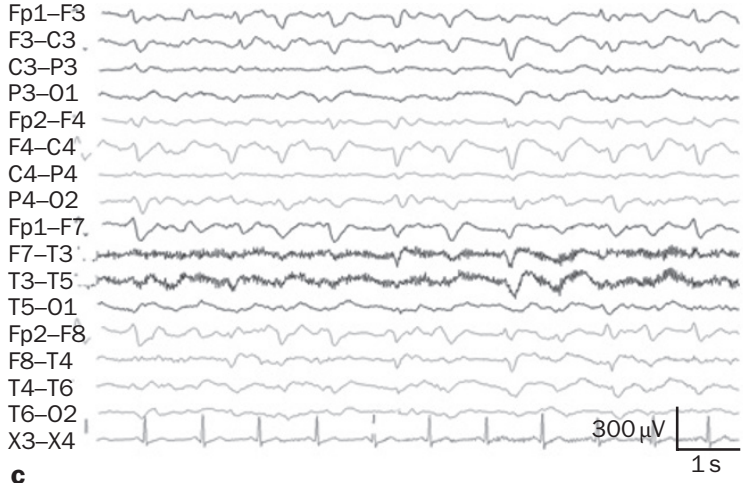

Fp1-F7

F7-T3

T3-T5

T5-01

Fp2-F8

F8-T4

T4-T6

$\mathrm{T} 6-02$

Fp1-F3

F3-C3

C3-P3

P3-01

Fp2-F4 2

F4-C4 2

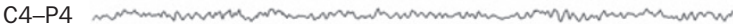

$\mathrm{P4}-02$

$\mathrm{X} 1-\mathrm{X} 2$

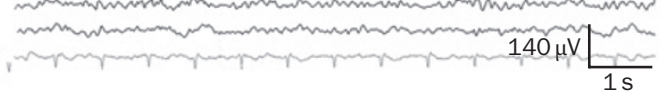

b

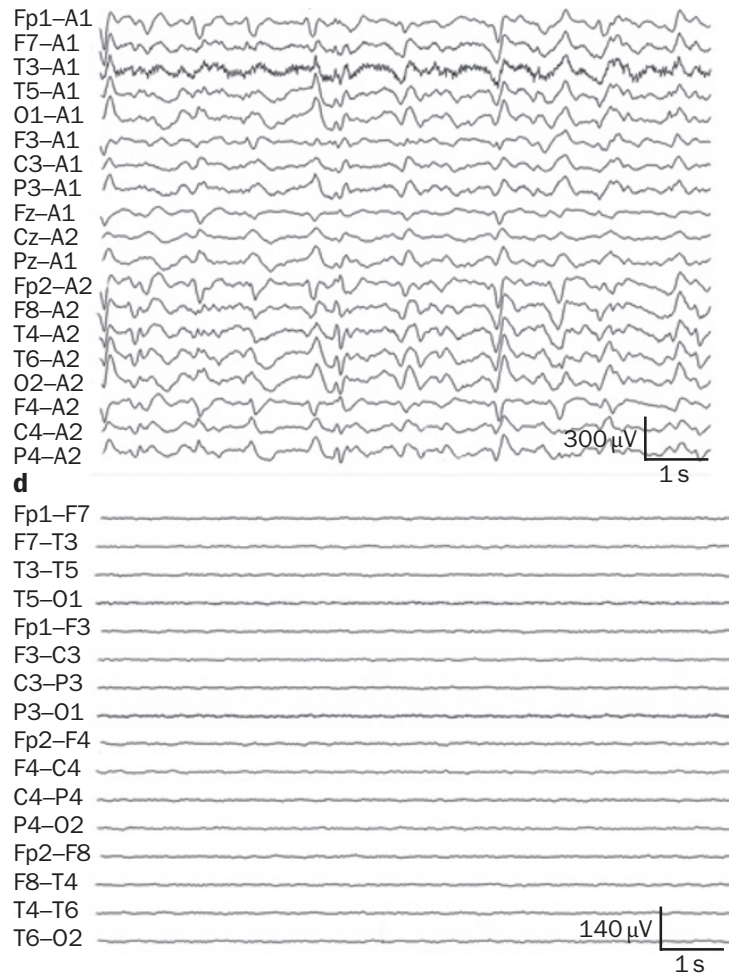

Figure 2 | EEG recordings of patients with encephalopathy. a | EEG recordings with bipolar longitudinal montage from a patient (56-year-old female) with encephalopathy, demonstrating triphasic waves. b | EEG recordings with a referential montage (ipsilateral ear reference) from the same patient as in (a), demonstrating triphasic waves. c| EEG recordings (with bipolar longitudinal montage) from a comatose patient (76-year-old male) with encephalopathy. A mixture of delta and theta frequency waveforms predominate. d | EEG recording (with bipolar longitudinal montage) from a comatose patient (50-yearold male) with encephalopathy. Generalized suppression is seen throughout all cerebral regions.

Neuroimaging in patients with SAE gives variable results. Some patients have normal brain MRI scans despite having SAE. In other patients, acute abnormalities are seen on MRI, including multiple ischaemic strokes or white matter lesions in the centrum semiovale (primarily at the level of Virchow-Robin spaces). ${ }^{23}$ The severity of these CNS lesions is associated with the severity of sepsis and correlates inversely with GCS scores. ${ }^{23}$ A neuroimaging study in critically ill patients with delirium (not specifically SAE) demonstrated an association between long duration of delirium and small brain volume at 3 months after hospital discharge. ${ }^{24}$ Small brain volume was also associated with increased cognitive impairment at 12 months after discharge from hospital. ${ }^{24}$ Further studies are required to determine whether small brain volume occurs before or as a consequence of delirium during critical illness.

In patients with sepsis, the EEG data show progressive slowing of brain activity with increasing severity of SAE.$^{10}$ Mild encephalopathy is associated with slowing of brain activity in the theta range and severe encephalopathy is usually associated with excessive delta waves and, less commonly, with a burst-suppression pattern of activity (Figure 2; Table 1). ${ }^{10} \mathrm{~A}$ triphasic wave pattern is seen on EEG in approximately $20 \%$ of patients with sepsis. ${ }^{10}$ Even in patients showing no clear encephalopathy (with relatively preserved cognition) on physical examination, but with laboratory evidence of bacteraemia, EEG reveals brain abnormalities in $50 \%$ of cases. ${ }^{10}$ These changes on EEG are resolved when sepsis is treated.

Mortality is increased in patients with severe abnormalities on EEG. ${ }^{10}$ In particular, patients who have triphasic wave or burst-suppression patterns on EEG have higher mortality rates than those with abnormal theta or delta wave patterns. ${ }^{10,25}$ Notably, these abnormal EEG findings are not only seen in patients with SAE, but are also frequently recorded in patients with other types of encephalopathy ${ }^{26}$ and, therefore, do not shed light on the aetiology of SAE. Nevertheless, they provide objective evidence of cerebral disturbance and its severity in SAE.

The clinical manifestations of SAE can be detected before presentation of strong evidence of sepsis or SIRS. In our experience, changes in cognitive or mental status that are associated with SAE can present in susceptible patients up to $36-48 \mathrm{~h}$ before other systemic symptoms of sepsis or SIRS become apparent. Such patients, who have an otherwise normal neurological examination and neuroimaging data and no other aetiology factors associated with coma, might show unexplained reductions in level of consciousness. However, functional neurological investigations, such as EEG recordings, can demonstrate changes suggestive of sepsis, including diffuse slowing of brain activity and abundant triphasic waves. Thus, we propose that SAE is an early feature of systemic infection 
that presents before the Bone et al. ${ }^{4}$ criteria for sepsis are satisfied.

Overall, SAE remains a diagnosis of exclusion. In patients presenting with symptoms suggestive of SAE, a rigorous investigation for other treatable aetiologies of encephalopathy, such as systemic organ dysfunction, stroke, intracranial haemorrhage, meningoencephalitis or other metabolic disturbance, should be undertaken to avoid overlooking the cause of encephalopathy.

\section{Pathophysiology}

The pathophysiology of SAE has not been established, but several likely mechanisms have been proposed. These mechanisms are not mutually exclusive and might be involved to varying degrees in different patients, often acting in concert or at various stages of the systemic illness. This section provides a brief overview of the processes that are thought to have a considerable role in the pathogenesis of SAE (Figure 3).

\section{Microscopic brain injury}

Specific neuropathological changes have been described in patients who died from septic shock. In a prospective postmortem study of patients with sepsis, cerebral lesions were reported which in one patient, were compatible with multifocal necrotizing leukoencephalopathy. ${ }^{27}$ Further prospective data show that cerebral changes associated with sepsis include evidence of cerebral ischaemia, haemorrhages and micro-abscesses (Figure 4). ${ }^{28}$

\section{BBB function and cerebral microcirculation}

Adequate function of the cerebral microcirculation is important for maintenance of normal cerebral function. Increasing evidence provides support for altered cerebral microcirculation during sepsis. This alteration probably has an important role in SAE pathogenesis. In a sheep model of peritonitis, Taccone et al..$^{29}$ showed that septic shock is associated with reductions in the total perfused cerebral vessel density and in the proportion of perfused small vessels in the brain. Similarly, in patients with sepsis, disturbances occur in cerebral autoregulation and in response of cerebral blood vessels to carbon dioxide concentration. ${ }^{30}$ Several studies of the effects of sepsis on carbon-dioxide-induced cerebral vasoreactivity have produced conflicting results. ${ }^{31-33}$ One group found that cerebral vasoreactivity was impaired in patients with sepsis, ${ }^{31}$ whereas others showed that sepsis did not have any significant effect on cerebral vascular function. ${ }^{32,33}$

Rat models of sepsis show increased permeability of the $\mathrm{BBB}$ in sepsis, as detected by increased horseradish peroxidase staining of CNS samples taken from septic rats, compared with controls. ${ }^{34}$ All the above-mentioned changes have been documented in patients with SAE, but not all patients with SAE have these abnormalities: only some, if any, of these changes might be found in a given patient.

Together with altered cerebral microcirculation, dysfunction of the BBB alters the extracellular milieu of the brain. Patients with sepsis have higher levels of protein in the CSF than do control patients without sepsis. ${ }^{35}$ Animal
Table 1 | Changes in EEG recordings in patients with SAE*

\begin{tabular}{llllll}
\hline \multirow{2}{*}{$\begin{array}{l}\text { Degree of } \\
\text { encephalopathy }\end{array}$} & \multicolumn{5}{c}{ EEG findings (\% of patients) } \\
\cline { 2 - 6 } & Normal & $\begin{array}{l}\text { Theta } \\
\text { waves }\end{array}$ & $\begin{array}{l}\text { Delta } \\
\text { waves }\end{array}$ & $\begin{array}{l}\text { Triphasic } \\
\text { waves }\end{array}$ & $\begin{array}{l}\text { Burst-suppression } \\
\text { pattern }\end{array}$ \\
\hline None & 50 & 38 & 12 & 0 & 0 \\
Mild & 0 & 47 & 54 & 0 & 0 \\
Severe & 0 & 10 & 40 & 20 & 30 \\
\hline
\end{tabular}

*Generated from data provided by Young et al. ${ }^{86}$ Abbreviation: SAE, sepsis-associated encephalopathy.

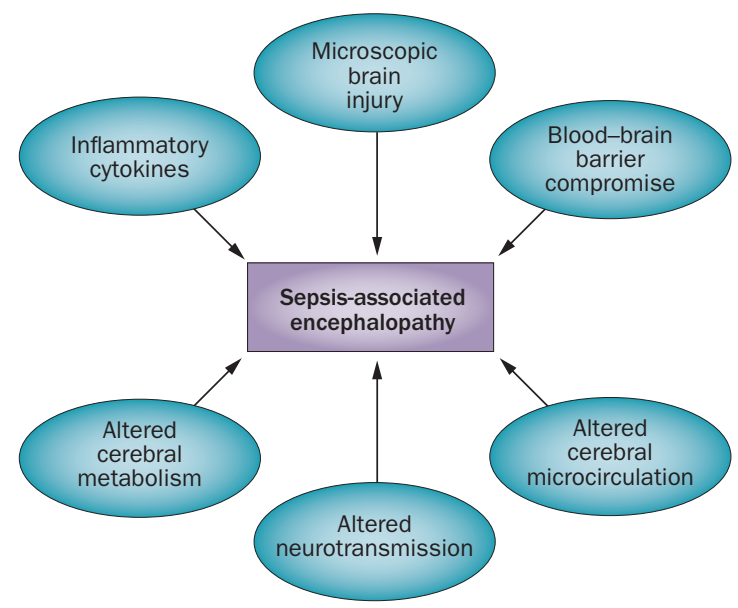

Figure 3 | Aetiology of SAE. The aetiology of SAE is multifactorial. Factors that contribute to SAE pathogenesis are shown in circles. The role of each factor varies from patient to patient and depends on the clinical situation. Abbreviation: SAE, sepsis-associated encephalopathy.

models of sepsis have demonstrated astrocytic dysfunction with detachment of astrocytes from the microvasculature. ${ }^{36,37}$ Taken together, altered blood flow at the microvascular level and BBB dysfunction affect cerebral function and probably contribute to the development and progression of SAE.

\section{Neurotransmission and cerebral metabolism}

Inflammatory and metabolic changes have been proposed to be associated with sepsis and to lead to alterations in cerebral neurotransmission. ${ }^{38,39}$ Van Gool et al..$^{38}$ proposed that cytokines such as tumour necrosis factor that are produced systemically during sepsis activate cerebral microglia which, in turn, release inflammatory mediators within the CNS. ${ }^{38}$ These inflammatory mediators cause aberrant neuronal function and, thereby, delirium and SAE.

Cholinergic neurotransmission has a role in this inflammatory process and can usually reduce the duration of delirium. ${ }^{38}$ If cholinergic function is impairedfor example, by neurodegenerative disease, pre-existing cholinergic dysfunction or anticholinergic medications -a more severe and long-lasting delirium could result. ${ }^{38}$ Conversely, a double-blind, placebo-controlled trial showed that delirium lasts longer and mortality is increased in patients treated with the cholinesterase inhibitor rivastigmine, compared with the control group, who did not have delirium. ${ }^{40}$ Given these findings, the 


\section{REVIEWS}
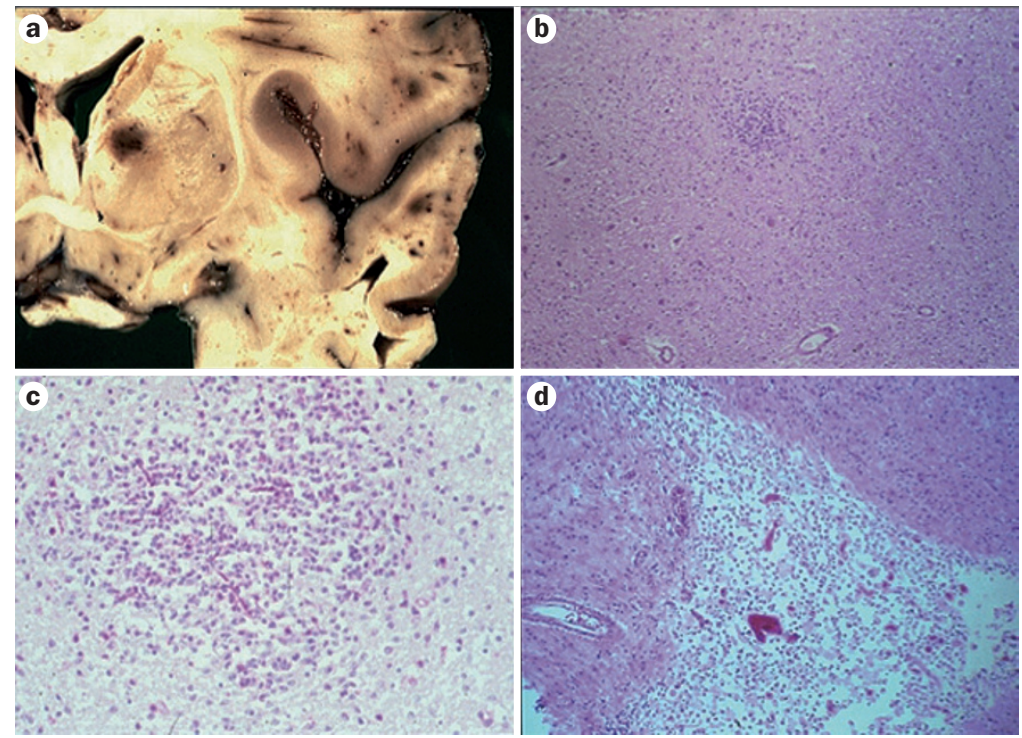

Figure 4 | Histological changes in the brains of patients with sepsis. a | Coronal section of the brain from a patient who died of sepsis. This section demonstrates evidence of cerebral ischaemia and small areas of haemorrhage. $\mathbf{b}$ | Areas of microabscess are seen on histological preparation of the brain from the patient in (a), with magnification of $\times 40$. c | The histopathological section in (b) visualized with a higher magnification ( $\times 200)$. d | Regions of cerebral ischaemia seen on the same histological preparation as in (a) and (b), with magnification of $\times 200$. Photos courtesy of D. Ramsay, London Health Sciences Centre and University of Western Ontario.

hypothesis that disturbance of neurotransmitter function is an aetiological factor for delirium should be more critically assessed in the future. The failure of rivastigmine to improve patient outcomes does not rule out the possibility that disturbances in cholinergic function contribute to development of delirium.

In studies of sepsis, IL-1 $\beta$ levels are increased in vitro and are associated with hippocampal dysfunction in rats. ${ }^{41}$ Examination of CSF proteins in patients with sepsis and delirium suggests that plasma concentrations of essential amino acid precursors (such as tryptophan and tyrosine) for synthesis of neurotransmitters, including serotonin, dopamine and noradrenaline, are abnormal (considerably higher or lower than normal levels) during sepsis. ${ }^{42}$ However, the same study showed that phenylalanine levels were normal in these patients. ${ }^{42}$

The role of altered levels of serum amino acids in sepsis and implications for possible neurotransmitter imbalance in the brain is complicated, controversial and still unclear. One study demonstrated an increase in levels of aromatic amino acids compared with levels of branched-chain amino acids in the plasma of patients with SAE ${ }^{43}$ Subsequently, a similar small observational study showed that only phenylalanine levels were increased in the plasma and CSF of patients with SAE. ${ }^{44}$ In addition to abnormal neurotransmission during the acute period of sepsis, long-term effects on neurotransmission have been shown. In a rat model of sepsis, animals that recovered showed loss of neurons in the hippocampus and the prefrontal cortex and decreased cholinergic innervation of the postrolandic cortical areas. ${ }^{45}$ These changes result in behavioural effects that persist despite resolution of sepsis. Clinical evidence for effects of SAE on long-term cognitive function also exists, as discussed below.

In sepsis, cerebral metabolism is altered. Experimental evidence shows that oxidative stress occurs in the early stages of sepsis, which results in decreased ATP synthesis. ${ }^{46}$ One study showed an increase in the ratio of superoxide dismutase to catalase activity in rats during early stages of sepsis ${ }^{47}$ and another study showed evidence of mitochondrial dysfunction during sepsis in mice. ${ }^{48}$ Some evidence from animal and tissue culture models shows sepsis-associated effects on the endothelium, which are probably caused by increased superoxide dismutase activity and reduced expression of endothelial nitric oxide synthase. ${ }^{49}$ Coagulation is activated in microvascular beds, leading to tissue ischaemia, and increased nitric oxide production has been proposed to damage mitochondria. ${ }^{49}$ Abundant studies in various animal models of sepsis support mitochondrial injury caused by oxidative stress. ${ }^{50,51}$ Although these studies have not focused on the brain, cerebral mitochondria are also likely to be negatively affected by sepsis, especially in the context of increased BBB permeability.

In a rat model of sepsis, alterations were also shown in cerebral protein expression. Proteins related to cell structure, energy production, signalling and cell death (chaperonins, $78 \mathrm{kDa}$ glucose-regulated protein, and glial fibrillary acidic protein [GFAP]) can be downregulated $24 \mathrm{~h}$ after onset of sepsis. ${ }^{52}$ Results of a recent study in a rat model of sepsis investigating the role of hydrogen sulphide, a novel gaseous transmitter in the CNS, suggest that increased hydrogen sulphide synthesis occurs in CNS in the setting of sepsis and is associated with increased levels of inflammatory cytokines, including tumour necrosis factor and IL-1 $\beta .{ }^{53}$

\section{Serum biomarkers}

Several serum biomarkers are elevated in patients with septic shock. These biomarkers include procalcitonin, IL-6, NSE and S100b. ${ }^{54,55}$ The majority of studies investigating serum biomarkers for sepsis have been performed in patients with a diagnosis of sepsis, but not specifically SAE. One study investigated the levels of NSE, S100b and GFAP in paediatric cases of sepsis with encephalopathy. ${ }^{54}$ These markers are specific to the CNS and their presence in serum reflects CNS injury ${ }^{56}$ Children with sepsis had higher levels of serum NSE, S100b and GFAP than did controls, and the serum levels of both NSE and S100b were highest in children who did not survive sepsis. ${ }^{54}$ Similar findings have been demonstrated in adults, with $42 \%$ of 170 patients with sepsis showing an increase in S100b serum levels, and 53\% of patients showing an increase in serum NSE levels during the first $72 \mathrm{~h}$ after hospital admission. ${ }^{57}$ Presence of S100b in serum of the patients reflects glial cell injury and abnormal BBB function, whereas presence of NSE, an intraneuronal enzyme, reflects neuronal injury. All these markers have also been studied in comatose patients who have survived cardiac arrest, and in patients with traumatic brain injury. ${ }^{56,58,59}$

Biomarkers are potentially useful for identification of septic illness in general, but they have been considered 
Table 2 | Interventions for SAE

\begin{tabular}{|c|c|c|c|}
\hline Intervention & Study population & Rationale & Outcome \\
\hline $\begin{array}{l}\text { Branched-chain amino acid } \\
\text { infusions }^{43}\end{array}$ & $\begin{array}{l}\text { Patients } \\
\text { with SAE* }\end{array}$ & $\begin{array}{l}\text { To restore the amino acid balance in } \\
\text { the blood and the neurotransmitter } \\
\text { balance in the brain }\end{array}$ & $\begin{array}{l}\text { Improved serum amino acid } \\
\text { imbalance and led to recovery } \\
\text { from encephalopathy }{ }^{\ddagger}\end{array}$ \\
\hline $\begin{array}{l}\text { Glutamate-release inhibitor } \\
{\text { (riluzole })^{78}}\end{array}$ & $\begin{array}{l}\text { Rat model } \\
\text { of sepsis }\end{array}$ & To reduce excitotoxicity in the brain & $\begin{array}{l}\text { Reduced brain oedema, blood- } \\
\text { brain barrier permeability and } \\
\text { histological damage }\end{array}$ \\
\hline Antioxidant (ascorbate) $)^{79}$ & $\begin{array}{l}\text { Various animal } \\
\text { models of sepsis }\end{array}$ & $\begin{array}{l}\text { To reduce microvascular damage } \\
\text { caused by free radicals }\end{array}$ & $\begin{array}{l}\text { Improved capillary blood flow, } \\
\text { reduced vascular permeability } \\
\text { and improved arteriolar } \\
\text { responsiveness to drugs }{ }^{\S}\end{array}$ \\
\hline $\begin{array}{l}\text { Plasmapheresis or plasma } \\
\text { filtration absorption } \\
\text { Antagonists of cytokine receptors } \\
81\end{array}$ & $\begin{array}{l}\text { Patients } \\
\text { with sepsis }\end{array}$ & $\begin{array}{l}\text { To reduce levels of inflammatory } \\
\text { cytokines in the blood } \\
\text { To block cytokine receptors }\end{array}$ & $\begin{array}{l}\text { Improved survival in patients } \\
\text { treated with plasmapheresis } \\
\text { No improvement in survival with } \\
\text { cytokine receptor blockers } \$\end{array}$ \\
\hline Activated protein $\mathrm{C}^{82}$ & $\begin{array}{l}\text { Patients } \\
\text { with sepsis }\end{array}$ & To reduce clotting in microcirculation & $\begin{array}{l}\text { Worse outcome and more } \\
\text { bleeding in patients } \$\end{array}$ \\
\hline $\begin{array}{l}\beta 2 \text { adrenergic receptor agonists } \\
\text { (dopexamine) }^{83}\end{array}$ & Pig model of SAE & To reduce cerebral oedema & $\begin{array}{l}\text { Reduced perivascular oedema } \\
\text { in the brain§}\end{array}$ \\
\hline
\end{tabular}

to have insufficient specificity and sensitivity to be clinically useful in differentiating SAE from other encephalopathies. ${ }^{60}$ Serum NSE and S100b protein levels, however, are sometimes elevated in patients with SAE. ${ }^{3}$ The clinical importance of elevated serum levels of NSE and $\mathrm{S} 100 \mathrm{~b}$ in patients with SAE, and the associations of these markers with the severity of SAE, have been questioned, given that not all studies have shown consistent results. ${ }^{61}$

A study showed that increased serum levels of IL-8 were associated with delirium in patients with inflammation, whereas IL-10 and amyloid- $\beta(\mathrm{A} \beta)_{1-42}$ and $\mathrm{A} \beta_{1-40}$ levels were elevated in patients with noninflammatory delirium. ${ }^{62}$ Patients with inflammatory delirium, who later showed cognitive impairment, had acutely elevated $\mathrm{A} \beta$ levels. ${ }^{57}$ Systemic elevations in serum procalcitonin and IL- 6 levels are also seen in patients with severe sepsis. ${ }^{55}$ Neither procalcitnonin nor IL-6, however, is a specific biomarker for CNS injury. Taken together, the above-mentioned studies support the hypothesis that $\mathrm{SAE}$ is, in fact, a result of direct CNS injury.

\section{Treatment and management}

The mainstay of management of SAE hinges on early detection of delirium - which is often the first manifestation of sepsis-determination of the underlying cause, accurate and prompt treatment of the infection, and provision of supportive care. Because SAE is not a consequence of direct CNS infection, treatment focus remains an appropriate management of the systemic infection, sepsis and the sequelae of SIRS. ${ }^{63}$ Early detection of SAE, even before sepsis is diagnosed, is important in prompting early investigation for and treatment of infection, thereby avoiding substantial morbidity and mortality associated with advanced-stage SAE.

In the absence of an identified source or causative organism, broad-spectrum antiobiotics are necessary. A prescription of antibiotics covering both gram-negative and gram-positive bacteria, as well as a high degree of suspicion for fungal infection as a potential cause of SAE, is required. Once a causative organism has been identified, narrowing the spectrum of antibiotics is appropriate, keeping in mind the sensitivities of the organisms to the antibiotics and the local antibiotic resistance patterns. Symptomatic management of delirium (for example, with antipsychotic therapy) is also necessary. ${ }^{64}$

Sedative medication is a common confounder in assessment of neurological status of patients in ICU. Withdrawal of sedative treatment and judicious use of benzodiazepine and opiate antagonists (such as flumazenil or naloxone, respectively) can be helpful, but often cannot completely resolve delirium. Triphasic waves on EEG are not commonly found in drug-induced encephalopathy (although they might be seen with baclofen, lithium, levodopa and pentobarbital intoxication), ${ }^{65}$ but are features of septic and metabolic (especially hepatic or uraemic) encephalopathy. ${ }^{26}$ Somatosensory evoked potentials are usually more resistant than EEG patterns to sedative agents or other psychoactive medications. Management of delirium in patients with SAE requires judicious use of sedative drugs. In general, lorazepam, a highly potent benzodiazepine, should be avoided. In a placebo-controlled trial comparing the effect of dexmedetomidine to that of lorazepam on clinical outcome in sepsis, patients treated with dexmedetomidine had more encephalopathy-free days, shorter time on the ventilator and lower mortality than those treated with lorazapam. ${ }^{66}$

Supportive care of patients with SAE should probably include EEG monitoring to detect nonconvulsive seizures, although the incidence of these seizures in our prospective monitoring study was low (less than 10\%), with no patients qualifying as having status epilepticus. ${ }^{67}$ Thus, prophylactic use of antiepileptic drugs is not justified.

Although no definitive therapy exists for SAE, a number of interventions have been tried or suggested in humans and animal models of SAE (Table 2). More-direct 
therapies aimed at containing the CNS abnormalities, such as microcirculatory changes, altered BBB permeability and abnormal neurotransmission, will hopefully be developed in the future. These therapies would conceivably help to prevent permanent impairment of cognition and cerebral function following recovery from sepsis and SAE.

\section{Prognosis}

As discussed above, SAE is known to cause both acute and chronic changes in cognition. The most easily detected changes occur acutely and manifest as encephalopathy and delirium. Mounting evidence in both animal models and in human studies, however, suggests that substantial long-term cognitive sequelae are associated with SAE. Animal models have shown longterm changes in behaviour, learning and memory following SAE ${ }^{45,68}$ One study demonstrated altered behaviour and long-term memory deficits in rats in an open-field and radial maze test. ${ }^{45}$ This study also showed reduced cholinergic innervation of the postrolandic cortex, the hippocampus and the prefrontal cortex. ${ }^{45}$ The hippocampal neuronal loss was postulated to be the basis of the long-term memory deficits. In vitro findings of enhanced $\gamma$-aminobutyric acid-related activity and altered hippocampal neuronal membrane excitability following exposure to lipopolysaccharide-induced chronic inflammation in a rat model of SAE provides further evidence for hippocampal synaptic dysfunction in sepsis. ${ }^{41}$ These studies suggest that both neuronal loss and alterations in cholinergic signalling have a role in the long-term effects of SAE.

We previously discussed the impact of SAE on mortality in the ICU. ${ }^{11}$ In survivors, it is now well-established that sepsis can lead to long-term cognitive impairment ${ }^{69-74}$ and lower health-related quality of life. ${ }^{70}$ In particular, patients rated the quality of life as being lower in the domains of physical function, general health, vitality and social function. ${ }^{70}$ In a follow-up study of critically ill patients with acute respiratory distress syndrome 6 years after discharge from the ICU, $40 \%$ of patients had residual disability and $23 \%$ of patients had cognitive impairment (particularly in attention skills). ${ }^{75}$ All patients with cognitive impairment also had residual disability, whereas only a small proportion of cognitively intact patients had residual disability. The patients with cognitive impairment also had the lowest quality of life scores, especially in the domains of physical and social function. ${ }^{75}$

One study showed that, in addition to cognitive sequelae, at 1 year after discharge, $29 \%$ of survivors of acute respiratory distress syndrome had substantial levels of depression and anxiety. ${ }^{72}$ In this study, the most significant correlates of poor quality of life were length of stay in ICU and emotional morbidity..$^{72}$ In another study, it was found that survivors of severe sepsis had considerable social and emotional morbidity compared with an agematched and sex-matched group who had been admitted to special care units with myocardial infarction. ${ }^{76}$

The above-mentioned data were obtained in survivors of severe illness in the ICU, but not specifically in patients with SAE. Whether the additional presence or severity of SAE affects cognition and quality of life is not known. In a literature review pertaining to long-term cognitive sequelae of SAE, the authors concluded that further research is necessary to determine the pathophysiology of cognitive impairment following SAE. ${ }^{77}$ Increased knowledge will facilitate the development of targeted interventions that can be administered while the patient is in the ICU and might limit the long-term consequences of SAE on daily life.

\section{Conclusions}

In conclusion, we propose that SAE can be an early feature of infection in the body before the 'Bone et al.' criteria for sepsis are satisfied. Furthermore, as SAE does not require evidence of brain pathology, its earliest manifestations are similar to SIRS-related encephalopathy and are likely to be largely due to inflammatory mediators (cytokines). In cases of severe SAE, prompt initiation of effective therapy at an early stage should avoid serious morbidity and mortality. Late phases of SAE are probably heterogeneous in terms of involved mechanisms, and the resultant laboratory abnormalities, brain dysfunction and even structural lesions can be diverse, including ischaemic lesions, encephalopathy due to failure of other organs, haematological disorders (such as disseminated intravascular coagulation) with brain purpura, and the associated critical illness neuromyopathy.

SAE is a clinical syndrome that is prevalent in patients in ICU and carries high mortality in severe cases owing to its association with multiorgan failure. Moreover, survivors of severe SAE have high morbidity. The brain is not merely a passive bystander in a reversible septic illness, but is one of the organs profoundly affected in an early and progressive manner. Recognition of delirium in ICU patients as an early feature of SAE is important, and aggressive investigation and treatment of infection and associated systemic effects, such as hypotension, is the most effective strategy to reduce mortality and morbidity associated with the disease. More research and clinical trials are needed to develop stratgies to address SAE without inhibiting the immune response and aggravating systemic infection. Some promising avenues that might be considered are use of free radical scavengers, restoration of the amino acid balance in the blood, and monitoring of and treating microcirculatory abnormalities in the brain and other organs. Clearly, there is much work to be done.

\section{Review criteria}

We searched the MEDLINE, PubMed, Google Chrome and Cochrane Library databases using the following search terms: "sepsis-associated encephalopathy", "septic encephalopathy", "sepsis AND encephalopathy", "sepsis AND delirium", and "sepsis AND coma". The search was restricted to the past 20 years, but was not restricted to types of articles or language of publication. We also searched the reference lists of selected full-text reviews and original articles to identify further references. 
1. Young, G. B. et al. The encephalopathy associated with septic illness. Clin. Invest. Med. 13, 297-304 (1990).

2. Zhang, L. N. et al. Epidemiological features and risk factors of sepsis-associated encephalopathy in intensive care unit patients: 2008-2011. Chin. Med. J. (Engl.) 125, 828-831 (2012).

3. lacobone, E. et al. Sepsis-associated encephalopathy and its differential diagnosis. Crit. Care Med. 37, S331-S336 (2009).

4. Bone, R. C., Sprung, C. L. \& Sibbald, W. J. Definitions for sepsis and organ failure. Crit. Care Med. 20, 724-726 (1992).

5. Levy, M. M. et al. 2001 SCCM/ESICM/ACCP/ATS/ SIS International Sepsis Definitions Conference. Crit. Care Med. 31, 1250-1256 (2003).

6. Bleck, T. P. et al. Neurologic complications of critical medical illnesses. Crit. Care Med. 21 , 98-103 (1993).

7. Eidelman, L. A., Putterman, D., Putterman, C. \& Sprung, C. L. The spectrum of septic encephalopathy. Definitions, etiologies, and mortalities. JAMA 275, 470-473 (1996).

8. Ely, E. W. et al. Delirium as a predictor of mortality in mechanically ventilated patients in the intensive care unit. JAMA 291, 1753-1762 (2004).

9. Bolton, C. F., Young, G. B. \& Zochodne, D. W. The neurological complications of sepsis. Ann. Neurol. 33, 94-100 (1993).

10. Young, G. B., Bolton, C. F., Archibald, Y. M., Austin, T. W. \& Wells, G. A. The electroencephalogram in sepsis-associated encephalopathy. J. Clin. Neurophysiol. 9 , 145-152 (1992).

11. Sprung, C. L. et al. Impact of encephalopathy on mortality in the sepsis syndrome. The Veterans Administration Systemic Sepsis Cooperative Study Group. Crit. Care Med. 18, 801-806 (1990).

12. Salluh, J. I. et al. Delirium epidemiology in critica care (DECCA): an international study. Crit. Care 14, R210 (2010)

13. Wilson, J. X. \& Young, G. B. Progress in clinical neurosciences: sepsis-associated encephalopathy: evolving concepts. Can. J. Neurol. Sci. 30, 98-105 (2003).

14. MacKenzie, I. M. The haemodynamics of human septic shock. Anaesthesia 56, 130-144 (2001)

15. Douglas, V. C. \& Josephson, S. A. Altered mental status. Continuum (Minneap. Minn.) 17, 967-983 (2011)

16. Miu, D. K. \& Yeung, J. C. Incidence of poststroke delirium and 1-year outcome. Geriatr. Gerontol. Int. http://dx.doi.org/10.1111/ j.1447-0594.2012.00871.x.

17. Khan, B. A. et al. Delirium in hospitalized patients: implications of current evidence on clinical practice and future avenues for research - a systemic evidence review. J. Hosp. Med. http://dx.doi.org/10.1002/jhm.1949.

18. Inouye, S. K. et al. Clarifying confusion: the confusion assessment method. A new method for detection of delirium. Ann. Intern. Med. 113, 941-948 (1990).

19. Reade, M. C., Eastwood, G. M., Peck, L., Bellomo, R. \& Baldwin, I. Routine use of the Confusion Assessment Method for the Intensive Care Unit (CAM-ICU) by bedside nurses may underdiagnose delirium. Crit. Care Resusc. 13, 217-224 (2011)

20. van Eijk, M. M. et al. Routine use of the confusion assessment method for the intensive care unit: a multicenter study. Am. J. Respir. Crit. Care Med. 184, 340-344 (2011).

21. Tomasi, C. D. et al. Comparison of CAM-ICU and ICDSC for the detection of delirium in critically ill patients focusing on relevant clinical outcomes. J. Crit. Care 27, 212-217 (2012).

22. Wijdicks, E. F., Bamlet, W. R., Maramattom, B. V., Manno, E. M. \& McClelland, R. L. Validation of a new coma scale: The FOUR score. Ann. Neurol. 58, 585-593 (2005).

23. Sharshar, T. et al. Brain lesions in septic shock: a magnetic resonance imaging study. Intensive Care Med. 33, 798-806 (2007).

24. Gunther, M. L. et al. The association between brain volumes, delirium duration, and cognitive outcomes in intensive care unit survivors: The VISIONS cohort magnetic resonance imaging study. Crit. Care Med. 40, 2022-2032 (2012).

25. Oddo, M., Carrera, E., Claassen, J., Mayer, S. A. \& Hirsch, L. J. Continuous electroencephalography in the medical intensive care unit. Crit. Care Med. 37, 2051-2056 (2009).

26. Kaplan, P. W. The EEG in metabolic encephalopathy and coma. J. Clin. Neurophysiol. 21, 307-318 (2004).

27. Sharshar, T. et al. Multifocal necrotizing leukoencephalopathy in septic shock. Crit. Care Med. 30, 2371-2375 (2002).

28. Sharshar, T. et al. The neuropathology of septic shock. Brain Pathol. 14, 21-33 (2004).

29. Taccone, F. S. et al. Cerebral microcirculation is impaired during sepsis: an experimental study. Crit. Care 14, R140 (2010).

30. Taccone, F. S. et al. Cerebral autoregulation is influenced by carbon dioxide levels in patients with septic shock. Neurocrit. Care 12, 35-42 (2010).

31. Terborg, C. et al. Dysfunction of vasomotor reactivity in severe sepsis and septic shock. Intensive Care Med. 27, 1231-1234 (2001).

32. Matta, B. F. \& Stow, P. J. Sepsis-induced vasoparalysis does not involve the cerebral vasculature: indirect evidence from autoregulation and carbon dioxide reactivity studies. Br. J. Anaesth. 76, 790-794 (1996).

33. Fulesdi, B. et al. Cerebral vasoreactivity to acetazolamide is not impaired in patients with severe sepsis. J. Crit. Care 27, 337-343 (2012).

34. du Moulin, G. C., Paterson, D., Hedley-Whyte, J. \& Broitman, S. A. E. coli peritonitis and bacteremia cause increased blood-brain barrier permeability. Brain Res. 340, 261-268 (1985).

35. Davies, D. C. Blood-brain barrier breakdown in septic encephalopathy and brain tumours. J. Anat. 200, 639-646 (2002).

36. Papadopoulos, M. C. et al. Faecal peritonitis causes oedema and neuronal injury in pig cerebral cortex. Clin. Sci. (Lond.) 96, 461-466 (1999).

37. Nishioku, T. et al. Detachment of brain pericytes from the basal lamina is involved in disruption of the blood-brain barrier caused by lipopolysaccharide-induced sepsis in mice. Cell. Mol. Neurobiol. 29, 309-316 (2009).

38. van Gool, W. A., van de Beek, D. \& Eikelenboom, P. Systemic infection and delirium: when cytokines and acetylcholine collide. Lancet 375, 773-775 (2010).

39. Jacob, A., Brorson, J. R. \& Alexander, J. J. Septic encephalopathy: inflammation in man and mouse. Neurochem. Int. 58, 472-476 (2011).

40. van Eijk, M. M. et al. Effect of rivastigmine as an adjunct to usual care with haloperidol on duration of delirium and mortality in critically ill patients: a multicentre, double-blind, placebocontrolled randomised trial. Lancet $\mathbf{3 7 6}$, 1829-1837 (2010).

41. Hellstrom, I. C., Danik, M., Luheshi, G. N. \& Williams, S. Chronic LPS exposure produces changes in intrinsic membrane properties and a sustained IL-beta-dependent increase in
GABAergic inhibition in hippocampal CA1 pyramidal neurons. Hippocampus 15, 656-664 (2005)

42. Pandharipande, P. P. et al. Plasma tryptophan and tyrosine levels are independent risk factors for delirium in critically ill patients. Intensive Care Med. 35, 1886-1892 (2009).

43. Freund, H. R., Ryan, J. A. Jr \& Fischer, J. E. Amino acid derangements in patients with sepsis: treatment with branched chain amino acid rich infusions. Ann. Surg. 188, 423-430 (1978).

44. Mizock, B. A. et al. Septic encephalopathy. Evidence for altered phenylalanine metabolism and comparison with hepatic encephalopathy. Arch. Intern. Med. 150, 443-449 (1990).

45. Semmler, A. et al. Long-term cognitive impairment, neuronal loss and reduced cortical cholinergic innervation after recovery from sepsis in a rodent model. Exp. Neurol. 204, 733-740 (2007).

46. Azevedo, L. C. Mitochondrial dysfunction during sepsis. Endocr. Metab. Immune Disord. Drug Targets. 10, 214-223 (2010).

47. Barichello, T. et al. Oxidative variables in the rat brain after sepsis induced by cecal ligation and perforation. Crit. Care Med. 34, 886-889 (2006).

48. d'Avila, J. C. et al. Sepsis induces brain mitochondrial dysfunction. Crit. Care Med. 36 , 1925-1932 (2008).

49. Cepinskas, G. \& Wilson, J. X. Inflammatory response in microvascular endothelium in sepsis: role of oxidants. J. Clin. Biochem. Nutr. 42, 175-184 (2008)

50. Brealey, D. et al. Mitochondrial dysfunction in along-term rodent model of sepsis and organ failure. Am. J. Physiol. Regul. Integr. Comp. Physiol. 286, R491-R497 (2004).

51. Crouser, E. D., Julian, M. W., Blaho, D. V. \& Pfeiffer, D. R. Endotoxin-induced mitochondria damage correlates with impaired respiratory activity. Crit. Care Med. 30, 276-284 (2002)

52. Hinkelbein, J. et al. Alterations in cerebral metabolomics and proteomic expression during sepsis. Curr. Neurovasc. Res. 4, 280-288 (2007).

53. Chen, D. et al. Effects of hydrogen sulfide on a rat model of sepsis-associated encephalopathy. J. Huazhong Univ. Sci. Technolog. Med. Sci. 31, 632-636 (2011).

54. Hsu, A. A. et al. Neurological injury markers in children with septic shock. Pediatr. Crit. Care Med. 9, 245-251 (2008).

55. Fioretto, J. R. et al. Interleukin-6 and procalcitonin in children with sepsis and septic shock. Cytokine 43, 160-164 (2008).

56. Berger, R. P. et al. Serum neuron-specific enolase, S100B, and myelin basic protein concentrations after inflicted and noninflicted traumatic brain injury in children. J. Neurosurg. 103, 61-68 (2005)

57. Nguyen, D. N. et al. Elevated serum levels of S-100beta protein and neuron-specific enolase are associated with brain injury in patients with severe sepsis and septic shock. Crit. Care Med. 34, 1967-1974 (2006).

58. Lamers, K. J. et al. Protein S-100B, neuronspecific enolase (NSE), myelin basic protein (MBP) and glial fibrillary acidic protein (GFAP) in cerebrospinal fluid (CSF) and blood of neurological patients. Brain Res. Bull. 61, 261-264 (2003)

59. Wijdicks, E. F. et al. Practice parameter: prediction of outcome in comatose survivors after cardiopulmonary resuscitation (an evidence-based review): report of the Quality Standards Subcommittee of the American 


\section{REVIEWS}

Academy of Neurology. Neurology 67, 203-210 (2006).

60. Pierrakos, C. \& Vincent, J. L. Sepsis biomarkers: a review. Crit. Care 14, R15 (2010).

61. Piazza, O., Russo, E., Cotena, S., Esposito, G. \& Tufano, R. Elevated S100B levels do not correlate with the severity of encephalopathy during sepsis. Br. J. Anaesth. 99, 518-521 (2007).

62. van den Boogaard, M. et al. Biomarkers associated with delirium in critically ill patients and their relation with long-term subjective cognitive dysfunction; indications for different pathways governing delirium in inflamed and noninflamed patients. Crit. Care 15, R297 (2011)

63. Dellinger, R. P. et al. Surviving Sepsis Campaign: international guidelines for management of severe sepsis and septic shock: 2008. Intensive Care Med. 34, 17-60 (2008).

64. Markowitz, J. D. \& Narasimhan, M. Delirium and antipsychotics: a systematic review of epidemiology and somatic treatment options. Psychiatry (Edgmont) 5, 29-36 (2008).

65. Blume, W. T. Drug effects on EEG. J. Clin Neurophysiol. 23, 306-311 (2006).

66. Pandharipande, P. P. et al. Effect of dexmedetomidine versus lorazepam on outcome in patients with sepsis: an a priori-designed analysis of the MENDS randomized controlled trial. Crit. Care 14, R38 (2010).

67. Young, G. B. \& Doig, G. S. Continuous EEG monitoring in comatose intensive care patients: epileptiform activity in etiologically distinct groups. Neurocrit. Care 2, 5-10 (2005).

68. Barichello, T. et al. Long-term cognitive impairment in sepsis survivors. Crit. Care Med. 33, 1671 (2005).
69. Soriano, F. G. Quality of life: late sequela in sepsis. Crit. Care Med. 33, 262-263 (2005).

70. Heyland, D. K. \& Kutsogiannis, D. J. Quality of life following critical care: moving beyond survival. Intensive Care Med. 26, 1172-1175 (2000).

71. Gordon, S. M., Jackson, J. C., Ely, E. W., Burger, C. \& Hopkins, R. O. Clinical identification of cognitive impairment in ICU survivors: insights for intensivists. Intensive Care Med. $\mathbf{3 0}$ 1997-2008 (2004).

72. Hopkins, R. O., Weaver, L. K., Chan, K. J. \& Orme, J. F. Jr. Quality of life, emotional, and cognitive function following acute respiratory distress syndrome. J. Int. Neuropsychol. Soc. 10 1005-1017 (2004)

73. Hopkins, R. O. et al. Neuropsychological sequelae and impaired health status in survivors of severe acute respiratory distress syndrome. Am. J. Respir. Crit. Care Med. 160 50-56 (1999).

74. Hough, C. L. \& Curtis, J. R. Long-term sequelae of critical illness: memories and health-related quality of life. Crit. Care 9, 145-146 (2005)

75. Rothenhausler, H. B., Ehrentraut, S., Stoll, C., Schelling, G. \& Kapfhammer, H. P. The relationship between cognitive performance and employment and health status in long-term survivors of the acute respiratory distress syndrome: results of an exploratory study. Gen. Hosp. Psychiatry 23, 90-96 (2001).

76. Lazosky, A., Young, G. B., Zirul, S. \& Phillips, R. Quality of life after septic illness. J. Crit. Care 25, 406-412 (2010).

77. Comim, C. M. et al. Cognitive impairment in the septic brain. Curr. Neurovasc. Res. 6, 194-203 (2009).
78. Toklu, H. Z et al. The effects of riluzole on neurological, brain biochemical, and histological changes in early and late term of sepsis in rats. J. Surg. Res. 152, 238-248 (2009).

79. Wilson, J. X. Mechanism of action of vitamin C in sepsis: ascorbate modulates redox signaling in endothelium. Biofactors 35, 5-13 (2009).

80. Busund, R., Koukline, V., Utrobin, U. \& Nedashkovsky, E. Plasmapheresis in severe sepsis and septic shock: a prospective, randomised, controlled trial. Intensive Care Med. 28, 1434-1439 (2002).

81. Goldie, A. S. et al. Natural cytokine antagonists and endogenous antiendotoxin core antibodies in sepsis syndrome. The Sepsis Intervention Group. JAMA 274, 172-177 (1995).

82. Marti-Carvajal, A. J., Sola, I., Lathyris, D. \& Cardona, A. F. Human recombinant activated protein C for severe sepsis. Cochrane Database of Systematic Reviews, Issue 3. Art. No.: CD004388. http://dx.doi.org/10.1002/ 14651858.CD004388.pub5.

83. Moss, R. F., Parmar, N. K., Tighe, D. \& Davies, D. C. Adrenergic agents modify cerebral edema and microvessel ultrastructure in porcine sepsis. Crit. Care Med. 32, 1916-1921 (2004).

\section{Acknowledgements}

The authors thank D. Ramsay of the University of Western Ontario for providing the neuropathological images used in Figure 4.

\section{Author contributions}

Both authors made substantial contributions to researching data for article, discussion of content, writing, and reviewing and/or editing of the manuscript before submission. 